\title{
Association of Gender with Efficacy of Immunotherapy in Metastatic Melanoma
}

\author{
Varsha Jain', Sriram Venigalla', Kevin T. Nead', Wei-Ting Hwang², John N. Lukens', Tara C. Mitchell³, Jacob E. \\ Shabason ${ }^{1 *}$ \\ 'Department of Radiation Oncology, Perelman School of Medicine at the University of Pennsylvania, Philadelphia, Pennsylvania, USA. \\ ${ }^{2}$ Department of Biostatistics, Epidemiology and Informatics, Center for Clinical Epidemiology and Biostatistics, Perelman School of Medicine at the University of \\ Pennsylvania, Philadelphia, Pennsylvania, USA. \\ ${ }^{3}$ Division of Medical Oncology, Perelman School of Medicine at the University of Pennsylvania, Philadelphia, Pennsylvania, USA.
}

Article Info

\section{Article Notes}

Received: May 27, 2019

Accepted: July 15, 2019

\section{*Correspondence:}

Jacob E. Shabason, Department of Radiation Oncology, Perelman School of Medicine at the University of Pennsylvania, 3400 Civic Center Blvd, Philadelphia, PA 19104, USA; Telephone No: (215) 662-6515; Fax No: (215) 349-5445; Email: jacob.shabason@uphs.upenn.edu.

(c) 2019 Shabason JE. This article is distributed under the terms of the Creative Commons Attribution 4.0 International License.

\section{Keywords:}

Melanoma

Immunotherapy

Immune checkpoint inhibitors

Gender

\section{ABSTRACT}

Pre-clinical data from animal models suggest that the anti-tumor efficacy of immune checkpoint blockade agents may be influenced by gender specific sex hormones. However, recent meta-analyses of clinical data aimed at addressing the impact of gender on response to these agents have demonstrated conflicting results. Given the discordant evidence, we sought to evaluate the association of gender with the receipt and efficacy of modern immunotherapies in patients with metastatic melanoma. This retrospective cohort study used the National Cancer Database to identify patients who were $\geq 18$ years old with Stage IV melanoma from 2011 to 2015. Patterns of utilization of immunotherapy, including by gender, were assessed using multivariable logistic regression. A multivariable Cox proportional hazards model, including an interaction term between the receipt of immunotherapy and gender, was used to evaluate whether gender modified the association of receipt of immunotherapy with hazards of death. 11,944 patients met study inclusion criteria. Of these, $8,093(68 \%)$ were males and 3,851 (32\%) were females. 2,930 (25\%) patients received immunotherapy while $9,014(75 \%)$ did not. There was no statistically significant difference in the receipt of immunotherapy between males and females. On multivariable analysis, receipt of immunotherapy was associated with a survival benefit in both males and females. However, a statistically significant difference in efficacy of immunotherapy based on gender was not observed $\left(p_{\text {interaction }}=0.422\right)$. Utilizing a real world cohort of patients derived from a national cancer registry, gender was not associated with differences in immunotherapy survival outcomes in patients with metastatic melanoma.

\section{Introduction}

Modern immunotherapy agents targeting cellular immune checkpoint pathways have demonstrated promising survival outcomes in metastatic melanoma, and are now the standard of care $^{1,2}$. While these drugs hold the promise of offering durable tumor control, only a fraction of treated patients respond. Understanding the clinical and biological factors (such as gender) that may modulate response to these agents is imperative to better select the most efficacious therapies for a particular patient as well as to inform future clinical trials.

Pre-clinical data from animal models does suggest sex hormone based modulation of immune pathways ${ }^{3,4}$. However, recent metaanalyses evaluating gender-associated differences in response to modern immunotherapies in advanced cancers have shown conflicting results ${ }^{5-7}$. 
Given this area of clinical controversy, we evaluated a real world cohort of patients with metastatic melanoma derived from a national cancer registry to examine the association of gender with the receipt of and survival outcomes with immunotherapy.

\section{Methods}

This study was exempt from review by our institutional review board as we used a de-identified dataset. We included patients who were $\geq 18$ years, had Stage IV (metastatic) cutaneous melanoma, and were diagnosed from 2011- 2015. This time period was chosen given the approval of Ipilimumab, the first modern immune checkpoint inhibitor (ICI) for metastatic melanoma, in 2011. Patients were excluded if receipt of immunotherapy or the disease stage was unknown or missing (eFigure 1).

Baseline characteristics between patients who did and did not receive immunotherapy were compared using Pearson's $\chi^{2}$ test. A multivariable logistic regression model was utilized to evaluate the patterns of receipt of immunotherapy.

In order to determine if the association of receipt of immunotherapy with hazards of death was modified by gender, a multivariable Cox proportional hazards model which included an interaction term between receipt of immunotherapy (yes/no) and gender (male/female) was used. The same model as above was used to derive individual hazards of death associated with receipt of immunotherapy for males and females as listed in Table 3.

All baseline covariates (Table 1) were evaluated. Covariates achieving a threshold significance of $p<0.1$ on univariate analysis were included in multivariable logistic regression and Cox proportional hazards analyses (Table 2 and eTable 1, respectively).

Propensity score (PS) adjustment with robust variance estimation was used to further adjust for potential confounding factors ${ }^{8}$ and denoted the probability of

\section{Receipt of Immunotherapy}

Total, $\mathbf{n}$

Gender

Male

Female

Age

$18-49$

50-69

$\geq 70$

Race

Non-Hispanic White

Non-Hispanic Black

Hispanic

Other

Facility Area

Metropolitan

Urban

Rural

Unknown

Insurance

Commercial

Medicare

Medicaid

Uninsured

Other

Zip Code Education Level

$\geq 21 \%$

$13 \%-20.9 \%$

\section{No}

9,014

6,102

2,912

1,187

3,921

3,906

8,490

133

238

153

7,181

1,394

193

246

2,954

4,535

711

486

328

1,309

2,250

Table 1: Baseline patient characteristics

$\begin{array}{cccccc}\% & \text { Yes } & \% & \text { Total } & \% & \text { chi2 } \\ (75) & 2,930 & (25) & 11,944 & (100) & \end{array}$

\subsection{6}

$\begin{array}{lcccc}(68) & 1,991 & (68) & 8,093 \\ (32) & 939 & (32) & 3,851\end{array}$

(13) 558

(43) $\quad 1,473$

(43) $\quad 899$

(19)

(50)

(31)

1,745

5,394

4,805

(2)

(94)

(1)

(3)

2,767
35
70
58

(80)

(2)

(3)

(33)

(8)

(5)

(4)

2,427
369
50
84

1,314
1,208
194
105
109

(25)
(45)

(41)

(7)

(4)

(4)

300

690

(10)

(24)
(94)

(1)

(2)

(2)

(2)

(3)

11,257

168

308

211

9,608

1,763

243

330

4,268

5,743

905

591

437
$<0.001$

(15)

(40)

0.425

(94)

0.001

$<0.001$

$<0.001$ 


\begin{tabular}{|c|c|c|c|c|c|c|c|}
\hline $7 \%-12.9 \%$ & 3,083 & (34) & 1,032 & (35) & 4,115 & (34) & \\
\hline$<7 \%$ & 2,345 & (26) & 903 & (31) & 3,248 & (27) & \\
\hline Unknown & 27 & $(<1)$ & 5 & $(<1)$ & 32 & $(<1)$ & \\
\hline Zip Code Median Income & & & & & & & $<0.001$ \\
\hline$<38,000$ & 1,311 & (15) & 337 & $(12)$ & 1,648 & (14) & \\
\hline $38,000-47,999$ & 2,168 & (24) & 612 & $(21)$ & 2,780 & (23) & \\
\hline $48,000-62,999$ & 2,512 & $(28)$ & 857 & (29) & 3,369 & (28) & \\
\hline$\geq 63,000$ & 2,983 & (33) & 1,119 & (38) & 4,102 & (34) & \\
\hline Unknown & 40 & $(<1)$ & 5 & $(<1)$ & 45 & $(<1)$ & \\
\hline Facility Type & & & & & & & $<0.001$ \\
\hline Non-Academic & 5,367 & $(60)$ & 1,297 & (44) & 6,664 & (56) & \\
\hline Academic & 3,215 & (36) & 1,404 & (48) & 4,619 & (39) & \\
\hline Unknown & 432 & (5) & 229 & (8) & 661 & $(6)$ & \\
\hline Facility Location & & & & & & & $<0.001$ \\
\hline East & 1,591 & (18) & 622 & $(21)$ & 2,213 & (19) & \\
\hline South & 3,317 & (37) & 880 & (30) & 4,197 & (35) & \\
\hline Central & 2,042 & (23) & 669 & (23) & 2,711 & (23) & \\
\hline West & 1,632 & (18) & 530 & (18) & 2,162 & (18) & \\
\hline Unknown & 432 & (5) & 229 & (8) & 661 & $(6)$ & \\
\hline Charlson Deyo Score & & & & & & & $<0.001$ \\
\hline 0 & 6,662 & (74) & 2,410 & $(82)$ & 9,072 & (76) & \\
\hline 1 & 1,608 & (18) & 398 & (14) & 2,006 & (17) & \\
\hline 2 & 484 & (5) & 91 & (3) & 575 & (5) & \\
\hline 3 & 260 & (3) & 31 & $(1)$ & 291 & $(2)$ & \\
\hline Surgery to Metastatic site & & & & & & & 0.03 \\
\hline No & 6,542 & (73) & 2,068 & (71) & 8,610 & $(72)$ & \\
\hline Yes & 2,404 & (27) & 829 & $(28)$ & 3,233 & (27) & \\
\hline Unknown & 68 & (1) & 33 & $(1)$ & 101 & $(1)$ & \\
\hline Brain Metastasis & & & & & & & $<0.001$ \\
\hline None & 4,544 & $(50)$ & 1,480 & (51) & 6,024 & $(50)$ & \\
\hline Present & 2,425 & (27) & 632 & $(22)$ & 3,057 & (26) & \\
\hline Unknown & 2,045 & (23) & 818 & (28) & 2,863 & (24) & \\
\hline Liver Metastasis & & & & & & & $<0.001$ \\
\hline None & 5,441 & $(60)$ & 1,605 & (55) & 7,046 & (59) & \\
\hline Present & 1,474 & (16) & 505 & (17) & 1,979 & (17) & \\
\hline Unknown & 2,099 & (23) & 820 & (28) & 2,919 & (24) & \\
\hline Lung Metastasis & & & & & & & $<0.001$ \\
\hline None & 4,036 & $(45)$ & 1,111 & (38) & 5,147 & $(43)$ & \\
\hline Present & 2,893 & $(32)$ & 999 & (34) & 3,892 & (33) & \\
\hline Unknown & 2,085 & (23) & 820 & (28) & 2,905 & (24) & \\
\hline Bone Metastasis & & & & & & & $<0.001$ \\
\hline None & 5,646 & (63) & 1,697 & (58) & 7,343 & $(61)$ & \\
\hline Present & 1,287 & (14) & 416 & (14) & 1,703 & (14) & \\
\hline Unknown & 2,081 & (23) & 817 & (28) & 2,898 & (24) & \\
\hline Receipt of Chemotherapy & & & & & & & $<0.001$ \\
\hline No & 6,094 & (68) & 2,549 & (87) & 8,643 & $(72)$ & \\
\hline Yes & 2,620 & (29) & 336 & (11) & 2,956 & (25) & \\
\hline
\end{tabular}




\begin{tabular}{|c|c|c|c|c|c|c|c|}
\hline Unknown & 300 & (3) & 45 & $(2)$ & 345 & (3) & \\
\hline Receipt of Radiotherapy & & & & & & & $<0.001$ \\
\hline No & 5,778 & (64) & 1,740 & (59) & 7,518 & (63) & \\
\hline Yes & 3,166 & (35) & 1,184 & $(40)$ & 4,350 & (36) & \\
\hline Unknown & 70 & $(1)$ & 6 & $(<1)$ & 76 & $(1)$ & \\
\hline Year of Diagnosis & & & & & & & $<0.001$ \\
\hline 2011 & 1,859 & $(21)$ & 341 & $(12)$ & 2,200 & (18) & \\
\hline 2012 & 1,811 & (20) & 356 & $(12)$ & 2,167 & (18) & \\
\hline 2013 & 1,881 & $(21)$ & 559 & (19) & 2,440 & (20) & \\
\hline 2014 & 1,801 & (20) & 707 & (24) & 2,508 & $(21)$ & \\
\hline 2015 & 1,662 & (18) & 967 & (33) & 2,629 & $(22)$ & \\
\hline
\end{tabular}

Table 2: Adjusted odds associated with the receipt of immunotherapy in patients with metastatic melanoma

Adjusted Odds ratio

\section{Gender}

Male

Female

Age

Facility Area

Metropolitan

Urban

Rural

Unknown

Insurance

Commercial

Medicare

Medicaid

Uninsured

Other

\section{Zip Code Education Level}

$\geq 21 \%$

$13 \%-20.9 \%$

$7 \%-12.9 \%$

$<7 \%$

\section{Zip Code Median Income}

\section{$<38,000$}

38,000-47,999

48,000-62,999

$\geq 63,000$

\section{Facility Type}

Non-Academic

$$
\text { [reference] }
$$

0.92

0.96

[reference]

[reference]

0.97

0.54

0.49

0.79

[reference]

[reference]

[reference]
0.83

1.01

0.077

0.96

0.97

$<0.001$

$\begin{array}{llll}0.74 & 0.64 & 0.87 & <0.001 \\ 0.74 & 0.52 & 1.05 & 0.095 \\ 0.93 & 0.71 & 1.23 & 0.619\end{array}$

0.85

1.11

0.672

0.45

0.66

$<0.001$

0.38

0.62

$<0.001$

0.61

1.02

0.075

$\begin{array}{llll}1.31 & 1.10 & 1.55 & 0.002 \\ 1.30 & 1.08 & 1.55 & 0.005 \\ 1.41 & 1.15 & 1.72 & 0.001\end{array}$

$\begin{array}{llll}1.00 & 0.84 & 1.19 & 0.989 \\ 1.07 & 0.90 & 1.28 & 0.457 \\ 1.08 & 0.89 & 1.32 & 0.429\end{array}$




\section{Facility Location}

East

South

Central

West

\section{Distance from Treatment Facility}

$\leq 40$ miles

$>40$ miles

Unknown/Missing

\section{Charlson Deyo Score}

0

1

2

3

\section{Surgery to Metastatic Site}

No

Yes

Unknown

\section{Brain Metastasis}

None

Present

Unknown

\section{Liver Metastasis}

None

Present

Unknown

\section{Lung Metastasis}

None

Present

Unknown

Bone Metastasis

None

Present

Unknown

Receipt of Chemotherapy

No

Yes

Unknown

\section{[reference]}

$\begin{array}{llll}0.83 & 0.72 & 0.95 & 0.006 \\ 0.95 & 0.82 & 1.09 & 0.452 \\ 0.99 & 0.85 & 1.16 & 0.937\end{array}$

[reference]

[reference]

$$
\begin{aligned}
& 0.77 \\
& 0.57 \\
& 0.34
\end{aligned}
$$

[reference]

$$
0.95
$$

1.35

0.85

[reference]

[reference]

$$
1.58
$$

[reference]

$$
0.96
$$

[reference]

0.22

0.35 


$\begin{array}{lcccc}\text { Receipt of Radiation } & & & & \\ \text { No } & \text { [reference] } & & 1.74 & <0.001 \\ \text { Yes } & 1.56 & 1.40 & 1.00 & 0.050 \\ \text { Unknown } & 0.42 & 0.17 & \end{array}$

Year of Diagnosis

$\begin{array}{ccccc}2011 & \text { [reference] } & & & \\ 2012 & 1.05 & 0.88 & 1.24 & 0.610 \\ 2013 & 1.62 & 1.39 & 1.90 & <0.001 \\ 2014 & 2.22 & 1.91 & 2.59 & <0.001 \\ 2015 & 3.32 & 2.85 & 3.85 & <0.001\end{array}$

Table 3: Overall survival associated with immunotherapy in patients with metastatic melanoma ${ }^{1}$

\begin{tabular}{|ccccccc|}
\hline & \multicolumn{3}{c}{ Multivariable } & & \multicolumn{2}{c|}{ PS analysis } \\
Gender & HR $[95 \% \mathrm{Cl}]$ & $\mathbf{p}$-value & $\mathbf{P}_{\text {interaction }}$ & HR[95\% Cl] & p-value & $\mathbf{P}_{\text {interaction }}$ \\
Male & $0.56[0.52,0.61]$ & $<0.001$ & & $0.56[0.51,0.62]$ & $<0.001$ \\
Female & $0.62[0.55,0.70]$ & $<0.001$ & & $0.61[0.53,0.70]$ & $<0.001$ \\
& & & 0.422 & & 0.414 \\
\hline
\end{tabular}

$H R=$ Hazards Ratio, $\mathrm{Cl}=$ Confidence interval, $P S=$ Propensity Score

${ }^{1}$ Refer to etable 1 for a detailed list of covariates included in the multivariable model

receiving immunotherapy(matched for all covariates listed in eTable1). A two-tailed $p$-value $<0.05$ was considered statistically significant. Statistical analyses were performed using Stata SE, version 15.0 (StataCorp, College Station, TX).

\section{Results}

A total of 11,944 patients met study inclusion criteria. Of these, 2,930 (25\%) received immunotherapy while 9,014 $(75 \%)$ did not. There were 8,093 (68\%) males and 3,851 $(32 \%)$ females. The median age of the patient cohort was 66 years (range: 55-76 years). Detailed baseline patient characteristics are listed in Table 1.

$25 \%$ of the males and $24 \%$ of the females received immunotherapy. On multivariable logistic regression analysis, there was no statistically significant difference in the receipt of immunotherapy between males and females (OR: 0.92, 95\% CI: 0.83-1.01, p=0.077). Factors associated with an increased likelihood of receiving immunotherapy included treatment at academic centers, presence of extracranial metastatic disease (liver, lung or bone), and a later year of diagnosis. Conversely, factors associated with a decreased likelihood of receiving immunotherapy included having Medicaid and being uninsured (vs. commercial insurance), having a higher Charlson Deyo comorbidity score, receiving chemotherapy, and having brain metastases (Table 2).

On multivariable survival analysis, receipt of immunotherapy was associated with a survival benefit in both males (HR: 0.56, 95\% CI: 0.52-0.61, p<0.0001) and females (HR: 0.62, 95\% CI: 0.55-0.70, p<0.001). However, the interaction term testing whether the hazards of death associated with the receipt of immunotherapy is modified by gender was not significant $\left(\mathrm{p}_{\text {interaction }}=0.422\right)$; highlighting that there was no statistically significant difference in efficacy of immunotherapy based on gender (eTable 1). These findings were consistent on PS matched analysis (Table 3).

\section{Discussion}

We utilized the NCDB to examine the association between gender and receipt and efficacy of modern immunotherapies in a real world cohort of patients with metastatic melanoma. Our results show that there was no difference in the likelihood of receipt of immunotherapy between males and females. Additionally, while immunotherapy was associated with a significant survival benefit in both males and females, we did not observe a statistically significant difference in efficacy based on gender.

Consistent with the trend of increased adoption of ICIs for management of metastatic melanoma, we noted an increased likelihood of receiving immunotherapy in recent years ${ }^{9}$. We also observed that patients with brain metastases were less likely to receive immunotherapy as this cohort had been disproportionately excluded from initial melanoma clinical trials ${ }^{10}$. Lastly, disparities in adoption of modern therapies between academic vs. community centers has been well documented for other treatments ${ }^{11}$ and was also highlighted in our analyses. 
Recent research to evaluate if there is a meaningful clinical difference in response to ICIs by gender has resulted in conflicting results. Specifically, Conforti et $\mathrm{al}^{6}$ performed a meta-analysis of 20 prospective studies across numerous disease sites and found that males respond better than females when treated with both cytotoxic $\mathrm{T}$ lymphocyte associated protein-4 (CTLA-4) and programmed death protein-1 (PD-1) inhibitors. However, a recent metaanalysis published by Wallis et $\mathrm{l}^{5}$ failed to find a statistically significant association of gender with the efficacy of ICIs in multiple advanced cancers. Similar to the study by Wallis etal, our findings also suggest that the efficacy of immunotherapy does not differ on the basis of gender. Given the controversy in the aforementioned published meta-analyses of prospective clinical trials, our study contributes additional information regarding the association of gender with the efficacy of immunotherapy. Furthermore, our dataset derives from a large $(>10,000)$ heterogeneous sample of patients treated at more than 1,500 Commission on Cancer accredited facilities 12 across the United States, includes individual level patient data and simulates real life practice patterns.

\section{Limitations}

Our study has several key limitations. First, there is inherent selection bias given the retrospective nature of the analysis. To minimize this bias, we performed PS-weighted analysis to adjust for a range of measured confounders. Second, the NCDB does not specify the type of immunotherapy used and hence definitive conclusions about the agents that were administered such as therapies targeting CTLA-4 and PD-1/PD-L1, Interleukin-2, Interferon- $\alpha$ etc. cannot be drawn. Given the above limitation, we only included patients treated after the approval of the first ICI (ipilumumab) in 2011 for metastatic melanoma. Furthermore, the term immunotherapy as coded by the NCDB can refer to other antibody based therapies, but these are not approved for use in melanoma and therefore if present would only represent a small minority of patients. Lastly, we lacked information about dosage, treatment schedules, duration of treatment, and toxicity which may have contributed to the outcomes.

\section{Conclusions}

We demonstrate that in the era of ICIs, there does not appear to be a difference between men and women in the likelihood of receipt of immunotherapy for metastatic melanoma. Additionally, while the receipt of immunotherapy was associated with a survival benefit for both men and women, we did not observe a statistically significant difference in survival outcomes based on gender. These findings further inform the controversy regarding the efficacy of immunotherapy based on gender by contributing real world data from a national cancer registry. Future and ongoing clinical trials utilizing ICIs and other immune modulating agents should further evaluate gender specific responses.

\section{References}

1. Wolchok JD, Chiarion-Sileni V1, Gonzalez R, et al. Overall Survival with Combined Nivolumab and Ipilimumab in Advanced Melanoma. N Engl J Med. 2017; 377(14): 1345-1356. doi:http://dx.doi.org/10.1056/ NEJMoa1709684

2. Hodi FS, O'Day SJ, McDermott DF, et al. Improved Survival with Ipilimumab in Patients with Metastatic Melanoma. N Engl J Med. 2010; 363(8): 711-723. doi:10.1056/NEJMoa1003466

3. Polanczyk MJ, Hopke C, Vandenbark AA, et al. Treg suppressive activity involves estrogen-dependent expression of programmed death-1 (PD-1). Int Immunol. 2007; 19(3): 337-343. doi:10.1093/ intimm/dxl151

4. Wang C, Dehghani B, Li Y, et al. Membrane Estrogen Receptor Regulates Experimental Autoimmune Encephalomyelitis through Upregulation of Programmed Death 1. J Immunol. 2009; 182(5): 32943303. doi:10.4049/jimmunol.0803205

5. Wallis CJ, Butaney M, Satkunasivam RS, et al. Association of Patient Sex With Efficacy of Immune Checkpoint Inhibitors and Overall Survival in Advanced Cancers A Systematic Review and Meta-analysis. JAMA Oncol. 2019. doi:10.1001/jamaoncol.2018.5904

6. Conforti F, Pala L, Bagnardi V, et al. Articles Cancer immunotherapy efficacy and patients ' sex : a systematic review and meta-analysis. Lancet Oncol. 2018; 19(6): 737-746. doi:10.1016/S14702045(18)30261-4

7. Botticelli A, Onesti CE, Zizzari I, et al. The sexist behaviour of immune checkpoint inhibitors in cancer therapy? Onco. 2017; 8(59): 9933699346.

8. Austin PC. An introduction to propensity score methods for reducing the effects of confounding in observational studies. Multivariate Behav Res. 2011; 46(3): 399-424. doi:10.1080/00273171.2011.568 786

9. Kather JN, Berghoff AS, Ferber D, et al. Large-scale database mining reveals hidden trends and future directions for cancer immunotherapy. Oncoimmunology. 2018; 7(7). doi:10.1080/2162402X.2018.1444412

10. Pasquali S, Chiarion-Sileni V, Rossi CR, et al. Immune checkpoint inhibitors and targeted therapies for metastatic melanoma: A network meta-analysis. Cancer Treat Rev. 2017; 54: 34-42. doi:10.1016/j. ctrv.2017.01.006

11. Ramalingam S, Dinan MA, Crawford J. Survival comparison in patients with Stage IV lung cancer in Academic versus Community Centers in the United States. J Thorac Oncol. 2018. doi:10.1016/j. jtho.2018.09.007

12. American College of Surgeons, American Cancer Society. National Cancer Database. National Cancer Database. https://www.facs.org/ quality-programs/cancer/ncdb. Published 2017. 